\title{
De vuelta a la clínica: sin justificación no existe pregunta de investigación que valga
}

\author{
Juan O. Talavera, ${ }^{1}$ Rodolfo Rivas-Ruiz, ${ }^{2}$ Marcela Pérez-Rodríguez, ${ }^{2}$ Ivonne Analí Roy-Garcia ${ }^{2}$ y \\ Lino Palacios-Cruz ${ }^{3}$ \\ ${ }^{1}$ Dirección de Enseñanza e Investigación, Centro Médico ABC; ${ }^{2}$ Instituto Mexicano del Seguro Social, Coordinación de Investigación en Salud, \\ Centro Médico Nacional Siglo XXI, Centro de Adiestramiento en Investigación Clínica; ${ }^{3}$ Secretaría de Salud, Instituto Nacional de Psiquiatría \\ "Dr. Ramón de la Fuente", Subdirección de Investigaciones Clínicas, Departamento Epidemiología Clínica. Ciudad de México, México
}

\begin{abstract}
Resumen
La formulación de una pregunta de investigación clínica requiere la concurrencia de experiencia clínica y conocimiento en metodología y estadística. Inicialmente, la pregunta de investigación debe contar con una estructura que deje claro qué se busca (consecuencia o desenlace), en quién (estado basal) y por acción de qué (maniobra). Posteriormente, su argumentación debe explorar cuatro aspectos: factibilidad y sensatez del cuestionamiento, ausencia de respuesta previa, relevancia de la respuesta a obtener y aplicabilidad. Una vez que estos aspectos han sido cubiertos en forma satisfactoria puede considerarse que la pregunta es "Clínicamente relevante", que es diferente a significancia estadística (la probabilidad de que el resultado se deba al azar y que no refleja la relevancia de la pregunta ni de los resultados). Nunca se debe olvidar que toda maniobra conlleva eventos adversos, que cuando son graves demeritan los buenos resultados. Es imperativo estimar la posible respuesta desde la estructura de la pregunta; la función de la investigación clínica es corroborar o rechazar una hipótesis, no probar empíricamente para ver qué resulta.
\end{abstract}

PALABRAS CLAVE: Justificación. Pregunta de investigación clínica. Relevancia.

\begin{abstract}
A clinical research question requires the concurrence of clinical experience and knowledge on methodology and statistics in that who formulates it. Initially, a research question should have a structure that clearly establishes what is that which is being sought (consequence or outcome), in whom (baseline status), and by action of what (maneuver). Subsequently, its reasoning must explore four aspects: feasibility and reasonableness of the questioning, lack of a prior answer, relevance of the answer to be obtained, and applicability. Once these aspects are satisfactorily covered, the question can be regarded as being "clinically relevant", which is different from being statistically significant, which refers to the probability of the result being driven by chance, which does not reflect the relevance of the question or the outcome. One should never forget that every maneuver entails adverse events that, when serious, discredit good results. It is imperative to have the possible answer estimated from within the structure of the question. The function of clinical research is to corroborate or reject a hypothesis, rather than to empirically test to find out what the outcome is.
\end{abstract}

KEY WORDS: Justification. Clinical research question. Relevance.

Correspondencia:

Juan O. Talaver

E-mail: jotalaverap@abchospital.com
Fecha de recepción: 21-12-2018

Fecha de aceptación: 16-01-2019

DOI:10.24875/GMM.19004942
Gac Med Mex. 2019;155:168-175

Disponible en PubMed www.gacetamedicademexico.com 
Sabe más un burro preguntando, que un sabio contestando.

Sin pregunta no hay respuesta y sin justificación no hay respuesta que valga.

DichOS POPULARES

\section{Introducción}

Una pregunta de investigación clínica relevante es la que busca mejorar la salud o calidad de vida de los pacientes. Para contar con ella será necesario tener un cuestionamiento estructuralmente justificado.

Si la justificación que da lugar a la pregunta no es adecuada, se invertirá un gran esfuerzo con mínimos beneficios para los pacientes y, peor aún, en ocasiones, se expondrá a muchas personas a procedimientos o maniobras injustificadas.

La generación, publicación y uso inadecuado de información que responde a una pregunta injustificada $\sin$ duda es responsabilidad compartida de varios actores:

- Los investigadores, que en numerosas ocasiones inician un proyecto en el que ignoran la magnitud del beneficio esperado y la aplicabilidad del mismo o, peor aún, en ocasiones desde la planeación del estudio prevén un mínimo beneficio o inaplicabilidad del resultado y deliberadamente lo ignoran por un conflicto de intereses ajenos al bienestar de los pacientes o de la población. Aunado a esto, una vez que cuentan con los resultados y aprovechando la significancia estadística (que no es sinónimo de relevancia clínica), concluyen que la maniobra es útil, aun cuando el beneficio en la práctica clínica sea marginal o con altos costos y eventos adversos que superan el beneficio. ${ }^{1-4}$

- Los comités de ética e investigación, que aprueban la ejecución de proyectos solo con base en el cumplimiento de requerimientos éticos y científicos, sin que evalúen el valor social ${ }^{5}$ o el uso responsable de los recursos, siempre finitos. ${ }^{6}$

- Los comités editoriales de las publicaciones, que permiten la publicación con lenguaje tendencioso y conclusiones injustificadas o magnificadas. ${ }^{7,8}$

- Los médicos y autoridades de salud en la comunidad, quienes hacen uso indiscriminado de recomendaciones tan solo por su significancia estadística, sin reflexionar en el beneficio real esperado e, incluso, en ocasiones las agregan a guías de práctica clínica simplificadas, que indirectamente promueven el "consumo de información" sin cuestionamientos. ${ }^{9}$
Es así que todos los participantes, desde la generación de una propuesta de investigación hasta la utilización de los resultados, tenemos la obligación de contar con conocimiento y experiencia del tema de interés - preferentemente ser profesionales de la salud con experiencia clínica- y un mínimo de conocimiento en metodología y estadística, temas discutidos en 24 artículos de una serie titulada "Investigación clínica”, publicada de 2011 a 2014 en Revista Médica del Instituto Mexicano del Seguro Social, ${ }^{10,11}$ donde se buscó ofrecer al médico un instrumento para comprender cómo se genera e interpreta la información que utiliza en la atención que brinda día a día a sus pacientes y para orientar su quehacer en investigación clínica.

Si consideramos la concurrencia de experiencia clínica con un conocimiento elemental en metodología y estadística, entonces se puede juzgar la relevancia de la pregunta de investigación, que se genera por la combinación de una observación sistematizada durante el ejercicio de la medicina y un vacío en el conocimiento. Si la pregunta no tiene como base estos dos elementos, se corre el riesgo de contar con una pregunta injustificada y, en consecuencia, una respuesta irrelevante, no importando qué tan sofisticado sea el diseño metodológico o el análisis estadístico, ni cuánto dinero se invierta. ${ }^{12}$

\section{Justificación de la pregunta de investigación (Tabla 1)}

Inicialmente, la pregunta de investigación debe contar con una estructura que deje claro qué se busca (el desenlace o consecuencia esperada), en quién (el estado basal) y por acción de qué (la maniobra);10,13,14 este diseño arquitectónico de la investigación para estudios de causalidad fue descrito por Alvan R. Feinstein ${ }^{14}$ y constituye una descripción detallada del quehacer clínico con el cual todo médico en el área de la atención está familiarizado. Un ejemplo de pregunta clínica sería el siguiente: ¿en el paciente con diabetes mellitus tipo 2 con sobrepeso-obesidad (estado basal) será más relevante la reducción de peso (maniobra experimental) que el control farmacológico estricto de la glucosa (maniobra comparativa), para evitar el daño macro o microvascular (desenlace)?

Una vez que están claros los componentes de la pregunta - estado basal, maniobra y desenlace-, su argumentación obliga a explorar la factibilidad y sensatez del cuestionamiento generado por la observación, ${ }^{15}$ la ausencia de una respuesta previa, ${ }^{13,16}$ la 


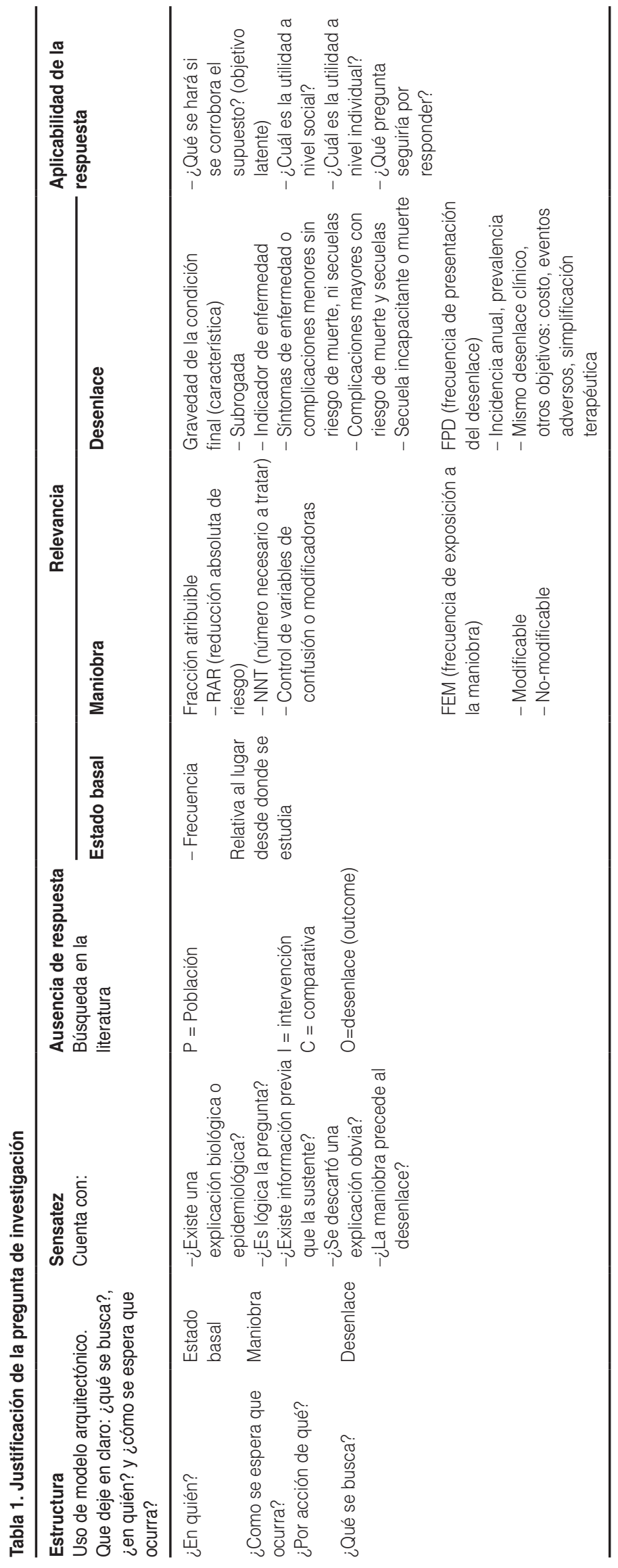


relevancia de la posible respuesta por obtener ${ }^{5,17}$ y su aplicabilidad..$^{15}$

\section{Factibilidad y sensatez del cuestionamiento generado a partir de una observación}

a) ¿La acción de la maniobra (observacional, por exposición a un factor de riesgo o protección, o experimental, por la asignación por parte del investigador) sobre el estado basal tiene una explicación biológica ${ }^{18} \mathrm{O}$ una base epidemiológica? No siempre es posible explicar los mecanismos biológicos por los cuales se observa una relación causal, dado que se depende del conocimiento disponible. Ejemplo: a fines del siglo XIX, Rigoni Stern (1842) analizó los datos de los certificados de defunción de las mujeres con cáncer cervicouterino; observó alta frecuencia de mujeres casadas, viudas y prostitutas y escasa frecuencia de mujeres núbiles y monjas. Concluyó que el desarrollo de este cáncer podría estar asociado con el contacto sexual, conocimiento que fue el precedente de lo que ahora sabemos sobre el papel del virus del papiloma humano en el desarrollo de cáncer cervicouterino. ${ }^{19}$

b) ¿Es lógica la explicación por la cual se justifica la probable acción de la maniobra? Ejemplo: el mecanismo por el cual la infección por el virus del papiloma humano se asocia con el desarrollo de cáncer cervicouterino, entre otras razones, se explica a través de las proteínas víricas E6, E7 y pRb, que condicionan la perpetuación de las células a través de la inactivación del supresor tumoral p53. Este y otros mecanismos provocan la proliferación celular con una menor capacidad de reparación de las mutaciones, que finalmente lleva al desarrollo de cáncer. ${ }^{20}$

c) ¿Las observaciones que dan origen a la pregunta provienen de evidencia directa y justificada y no de una suposición o información aún no validada? En ese caso no se justificaría una segunda suposición. Ejemplo: tras la epidemia de influenza en 2009 se publicaron estudios en los que se planteaba a la obesidad como factor de riesgo para el desarrollo de complicaciones y mortalidad. Aun cuando no se había demostrado la consistencia de estos resultados, se llevaron a cabo investigaciones para encontrar los mecanismos fisiopatológicos que explicaran esta supuesta asociación. ${ }^{21}$ Posteriormente se demostró que al ajustar por la presencia de otras comorbilidades se perdía el efecto de la obesidad. ${ }^{22}$

d) ¿Se ha descartado la existencia de una explicación alterna obvia, independiente de la maniobra de interés? Ejemplo: En 2009, en Nature se publicó un artículo que evaluaba el efecto de la iluminación de la habitación en niños menores de dos años y su asociación con el desarrollo de miopía. Los niños que durmieron con la luz encendida tuvieron una mayor prevalencia de miopía (48 \%) que aquellos que durmieron con la luz apagada $(9 \%) .{ }^{23}$ En 2010, otros investigadores encontraron resultados diferentes; atribuyeron la diferencia a la falta de medición de una tercera variable: el antecedente de miopía de los padres, razón por la que se mantenía encendida la luz de la habitación. ${ }^{24}$

e) ¿La maniobra precede al desenlace? Es decir, siempre se observa que la acción de la maniobra sobre el estado basal antecede al efecto o desenlace. Bradford Hill afirmaba que esto es particularmente relevante en enfermedades de lento desarrollo y ejemplificaba con las siguientes preguntas: ¿puede una dieta particular llevar a tener una enfermedad " $X$ " o será que las etapas tempranas de la enfermedad provocan hábitos dietéticos peculiares? o ¿alguna ocupación en particular promueve la infección por el bacilo de la tuberculosis o será que los hombres y mujeres que seleccionan ese tipo de trabajo son más propensos a contraer tuberculosis, o será que de hecho ya habían contraído la enfermedad? ${ }^{18}$

\section{Ausencia de una respuesta previa}

La falta de respuesta previa deberá confirmarse a través de la búsqueda sistemática con el uso de la palabra PICO, acrónimo basado en los tres componentes de la pregunta: estado basal $(\mathrm{P}=$ population), maniobra ( $\mathrm{I}=$ intervention, $\mathrm{C}=$ comparative maneuver) $\mathrm{y}$ desenlace $(\mathrm{O}=$ outcome $),{ }^{13}$ lo cual no significa la ausencia de estudios previos sobre el tema; de hecho, la investigación busca resolver una necesidad a través de corroborar una hipótesis perfectamente documentada. Sin embargo, hay que cuidar que no se repita un proyecto de investigación para dar respuesta a lo ya contestado. Ejemplo: Fergusson et al. realizaron una revisión sistemática para evaluar la eficacia de la aprotonina en la disminución de sangrado perioperatorio. De 64 ensayos clínicos encontrados, en todos se indicaba que era un factor de protección; solo $20 \%$ mencionaba estudios previos. Lo anterior muestra un gran número de ensayos clínicos para evaluar una eficacia ya probada y la ausencia de referencia a los estudios que previamente la habían demostrado. ${ }^{25}$

\section{La relevancia de la posible respuesta}

Se ha considerado que una investigación no tiene valor social o científico cuando el conocimiento que 
pretende ser generado no contribuye a entender 0 resolver un problema de salud..$^{26}$ Así, mencionaremos la relevancia de la pregunta de investigación a partir de los tres componentes del modelo arquitectónico:

a) Relevancia de la condición basal. Se tiene que considerar la importancia por la ponderación de la frecuencia, que depende de si se realiza desde la toma de decisiones a nivel gubernamental o de los sistema de salud, o al interior de un hospital o en el consultorio. En el primer caso, resulta importante en patologías o condiciones de alta prevalencia o incidencia (como la diabetes mellitus, cuya prevalencia es de $9.4 \%) ; 27$ por su parte, las enfermedades raras puede ser de interés en centros de concentración. Ejemplo: la esclerosis múltiple tiene una prevalencia de 33 casos por 100000 habitantes en México, lo cual no justificaría su estudio en la población general, sin embargo, puede resultar relevante en un hospital de especialidades donde se tiene un registro de varios cientos de pacientes. ${ }^{28}$

b) Relevancia del desenlace. Entre las características importantes se encuentran:

- Frecuencia del desenlace. Mientras más frecuente es un desenlace desfavorable, mayor atención requiere. El ejemplo más sencillo es la obesidad infantil en México, la cual tiene un incremento que da a esta enfermedad dimensiones de problema de salud pública. ${ }^{29}$

- Gravedad de la condición final. Puede ordenarse de la menor a la mayor gravedad:

- Estudio de características subrogadas que no tienen traducción clínica. Ejemplo: los efectos antiinflamatorios de la saxagliptina en pacientes con diabetes mellitus tipo $2 .^{30}$

- Estudio de características propias de la enfermedad, pero sin manifestación ni consecuencia clínica. Ejemplo: estudio de la eficacia de la alogliptina versus glipizide en pacientes diabéticos tratados con metformina, donde el objetivo es el control de la glucemia sin explorar desenlaces clínicos. ${ }^{31}$

- Estudio de manifestaciones clínicas de la enfermedad o complicaciones menores generalmente reversibles, que no implican riesgo en la vida. Ejemplo: metaanálisis para evaluar el efecto del canaglifozin sobre la hemoglobina glucosilada, con beneficios en el peso y presión arterial. ${ }^{32}$

- Estudio de complicaciones mayores o irreversibles de la enfermedad, que pueden poner en riesgo la vida. Ejemplo: una cohorte de niños supervivientes de cáncer que recibieron quimioterapia muestra la presencia de sordera asociada con el tratamiento. ${ }^{33}$

- Estudio relacionado con mortalidad inmediata o mediata (siguientes cinco años) o secuelas incapacitantes. Ejemplo: estudio de recién nacidos prematuros cuyo objetivo fue identificar aquellos con mayor probabilidad de muerte 0 de hemorragia intraventricular grado IV. ${ }^{34}$

- Otras características importantes para el mismo desenlace clínico:

- Estudios de costo económico. Costo directo por la enfermedad o por tratamiento, como el costo por hospitalización, ${ }^{35} \mathrm{y}$ el costo indirecto, entre otros, al imposibilitar la realización de las actividades cotidianas. Ejemplo: costos indirectos atribuidos a complicaciones de la diabetes mellitus tipo 2, retinopatía USD $\$ 10323421$, enfermedad cardiovascular USD\$12 843134 , nefropatía USD $\$ 81 \quad 814 \quad 501$, neuropatía USD\$2 760 271, enfermedad vascular periférica USD\$2 $042601 .^{36}$

- Reducción de costos. Aún con el mismo éxito y eventos adversos, puede existir reducción de costos por disminución del precio o días de tratamiento. Ejemplo: se realizó un estudio sobre el desempeño de los datos clínicos y de las pruebas rápidas para el diagnóstico de influenza. Se encontró que si bien el uso de las pruebas rápidas representa un costo extra en la atención inicial de los pacientes con sospecha de influenza, al final disminuye los costos por un diagnóstico oportuno. ${ }^{37}$

- Simplificación terapéutica. Esta característica siempre impactará en el apego a la maniobra con el consecuente incremento en el éxito. Ejemplo: un estudio demostró que población pediátrica con neumonía adquirida en comunidad que tenía una dosis al día de antibiótico, presentó mayor apego, menores tasas de hospitalización y mayores tasas de curación, que aquella en la que se utilizaba un antibiótico de tres dosis. ${ }^{38}$

- Evaluación de eventos adversos. Toda maniobra tiene eventos adversos. Si bien estamos acostumbrados a observarlos en maniobras experimentales, de igual forma deben considerarse en las no experimentales (observacionales). Ejemplo: en el estudio ACCORD, se evaluó el efecto del control intensivo de la glucosa en pacientes diabéticos. El resultado fue que el 
control intensivo de la glucosa no disminuyó la mortalidad por infarto agudo del miocardio (HR $=0.97$, IC $95 \%=0.70-1.36$ ), pero sí se incrementó el porcentaje de pacientes con eventos de hipoglucemia severa de 6.94 a $21.6 \%$ (p $<0.03)^{39}$

- Balance beneficios versus eventos adversos. Cada vez que evaluamos el beneficio de una maniobra hay que restar los eventos adversos equivalentes. Ejemplo: en el estudio SPRINT, que analizó el efecto del control estricto de la hipertensión arterial comparado con el tratamiento estándar, se encontró que si bien a tres años se lograba disminuir $2.1 \%$ la enfermedad cardiovascular, la enfermedad renal se incrementó $3 \% .{ }^{40}$

c) Relevancia de la maniobra, para lo cual hay que considerar lo siguiente:

- La ganancia absoluta en el desenlace por acción de la maniobra - magnitud de la maniobra, con incremento o reducción absoluta del riesgo-. En general, para una maniobra terapéutica se acepta como clínicamente relevante una ganancia de $10 \%$ en forma directa, equivalente a un número necesario a tratar de 10. Sin embargo, hay que considerar beneficios aun menores cuando la maniobra es preventiva, de bajo costo y con riesgo mínimo o cuando el desenlace sea grave, deja secuelas incapacitantes o pone en riesgo la vida. Ejemplo: en un estudio se compararon comunidades que recibieron una intervención intensiva versus una intervención ocasional para disminuir la obesidad en niños; si bien el efecto fue marginal $\left(1.4 \mathrm{~kg} / \mathrm{m}^{2}\right)$, la intervención era de muy bajo costo y podía impactar a largo plazo. ${ }^{41}$

- El control de variables de confusión o modificadoras. Para que sea factible ponderar el valor real de una maniobra siempre deberá considerarse el beneficio atribuido a la misma una vez controladas las variables modificadoras y de confusión; de no ser así, en numerosas ocasiones se sobreestimará dicho beneficio. Ejemplo: ensayo clínico en pacientes obesos con diabetes mellitus tipo 2 cuyo objetivo fue el control glucémico al comparar tratamiento farmacológico versus tratamiento farmacológico + derivación gástrica o colocación de banda gástrica. A 12 meses los pacientes sometidos a procedimiento quirúrgico alcanzaron $38 \%$ de control glucémico contra $5 \%$ del grupo farmacológico, atribuyendo la mejoría únicamente a la maniobra quirúrgica, sin considerar la pérdida de peso durante el seguimiento como la principal variable de confusión. ${ }^{42}$ Otro ejemplo muy importante dado el impacto que ha representado en la medición del riesgo cardiovascular a nivel mundial es la omisión dentro del modelo de riesgo cardiovascular de Framingham, del antecedente de enfermedad cardiovascular, actividad física y obesidad, con lo cual se ha sobreestimado el de por sí mínimo riesgo que representa la elevación de lípidos, tensión arterial, glucosa y tabaquismo (0.4 a $0.6 \%$ anual). ${ }^{43}$

- La frecuencia de exposición a la maniobra. No todas las maniobras son acciones terapéuticas asignadas por el médico, como ocurre en un ensayo clínico; en consecuencia, a diferencia de este, donde en la mayoría de las ocasiones cada maniobra es distribuida a $50 \%$ de la población, en los estudios observacionales la exposición a la maniobra puede ser inferior, incluso por debajo de $10 \%$, lo cual tiene que considerarse aunado al impacto de la misma en el desenlace. Ejemplo: en un estudio para evaluar la asociación de niveles elevados de troponina con mortalidad intrahospitalaria en pacientes con falla cardiaca, solo $6.2 \%$ de los pacientes mostró elevación. ${ }^{44}$

- La presencia de maniobras modificables. Siempre es importante identificar factores de riesgo 0 pronóstico, pero resulta de mayor beneficio cuando son modificables. Ejemplo: la edad es un factor de riesgo no modificable tanto para osteoporosis como para enfermedad cardiovascular, no así el estilo de vida que siempre puede modificarse. ${ }^{45}$

\section{Aplicabilidad de la respuesta}

Desde el planteamiento y justificación de la pregunta tenemos que cuestionarnos la utilidad final y aplicabilidad de la posible respuesta. A esto se conoce como "objetivo latente". En consecuencia, al formular una hipótesis hay que imaginarse la respuesta y la aplicación de la misma. Cuando no tenemos claro el objetivo latente podemos perdernos en el camino y confundir el desenlace final con desenlaces intermedios, con lo que frecuentemente perdemos de vista la aplicabilidad de los resultados. Ejemplo: a través de diversas publicaciones se ha acumulado evidencia en torno a que los trastornos mentales tienen una base etiológica importante en el neurodesarrollo y en la neurobiología, ${ }^{46-48} \sin$ embargo, la aplicabilidad de este conocimiento con fines diagnósticos aún parece 
distante. De igual forma la exaltación de la dislipidemia, hipertensión y elevación de la glucosa como factores de riesgo cardiovascular ha provocado que gran cantidad de estrategias dirijan sus objetivos al control de estos factores y se ha descuidado el desenlace real al que deberían ir dirigidas.

\section{Conclusiones}

Una vez cubiertos en forma satisfactoria los aspectos de factibilidad y sensatez del cuestionamiento generado por la observación, ${ }^{15}$ ausencia de una respuesta previa, ${ }^{13,16}$ relevancia de la posible respuesta por obtener ${ }^{5,17}$ y aplicabilidad, puede concluirse que la pregunta es "clínicamente relevante".

Tal como en un proyecto de investigación desde el diseño se considera la probabilidad de contar con significancia estadística $(p<0.05)$-término que se refiere a la probabilidad de que el resultado se deba al azar-, de igual forma es indispensable justificar desde el inicio de la pregunta si esta es "clínicamente relevante". Ejemplo: al estudiar el efecto de las estatinas en la disminución de la mortalidad cardiovascular en hombres con dislipidemia, se observó una mortalidad de $10.3 \%$ a 10 años en el grupo placebo versus $8.6 \%$ en el grupo que recibió estatinas, con reducción relativa del riesgo de $18 \%(\mathrm{HR}=0.82$, IC $95 \%=0.69-0.96, p=0.02$ ). Si bien el resultado fue estadísticamente significativo, la magnitud del efecto de las estatinas sobre la mortalidad fue únicamente de $1.7 \%$ a 10 años $(0.17 \%$ al año, número necesario a tratar de 588); claramente la significancia estadística no es sinónimo de relevancia clínica. ${ }^{6}$

Adicionalmente, debemos mencionar que existen mediciones que si bien pueden representar en algunos proyectos el desenlace final, estamos obligados a considerarlas en todo proyecto; un ejemplo de esto es el control de calidad de la maniobra principal o la evaluación de los eventos adversos. Finalmente, debemos resaltar como mínimo un par de fundamentos éticos en la justificación de la pregunta: el uso responsable de recursos finitos y evitar la explotación de los sujetos de investigación. ${ }^{11}$

Obtener una respuesta adecuada para un cuestionamiento que no tiene relevancia clínica resulta sin sentido. Nunca debe olvidarse que toda maniobra conlleva eventos adversos, los cuales se deben restar al éxito cuando sean equivalentes o peores a este.

Es imperativo resaltar que cuando se estructura una pregunta se debe estimar la posible respuesta. La función de la investigación clínica es corroborar o rechazar la hipótesis generada a partir de una duda sin resolver, pero sustentada en información previa; de ninguna manera se trata de "ver qué pasa".

\section{Bibliografía}

1. Peto R, Gray R, Collins R, Wheatley K, Hennekens C, Jamrozik K, et al. Randomised trial of prophylactic daily aspirin in British male doctors. $\mathrm{Br}$ Med J (Clin Res Ed). 1988;296:313-316.

2. Steering Committee of the Physicians' Health Study Research Group. Final report on the aspirin component of the ongoing Physicians' Health Study. N Engl J Med.1989;321:129-135.

3. The Medical Research Council's General Practice Research Framework. Thrombosis prevention trial: randomised trial of low-intensity oral anticoagulation with warfarin and low-dose aspirin in the primary prevention of ischaemic heart disease in men at increased risk. Lancet. 1998; 351:233-241.

4. Action to Control Cardiovascular Risk in Diabetes Study Group, Gerstein HC, Miller ME, Byington RP, Goff DC, Bigger JT, et al. Effects of intensive glucose lowering in type 2 diabetes. $N$ Engl J Med. 2008;358:2545-2559.

5. Emanuel EJ, Wendler D, Grady C. What makes clinical research ethical? JAMA. 2000;283:2701-2711.

6. Ridker PM, Danielson E, Fonseca FA, Genest J, Gotto AM, Kastelein JJ, et al. Rosuvastatin to prevent vascular events in men and women with elevated C-reactive protein. N Engl J Med. 2008;359:2195-2207.

7. Buckley LF, Dixon DL, Wohlford GF, Wijesinghe DS, Baker WL, Van-Tassell BW. Intensive versus standard blood pressure control in SPRINT-Eligible Participants of ACCORD-BP. Diabetes Care. 2017;40:1733-1738.

8. Mi MY, Mukamal KJ. Comment on Buckley et al. Intensive versus standard blood pressure control in SPRINT-Eligible Participants of ACCORD-BP. Diabetes Care 2017;40:1733-1738. Diabetes Care. 2018; 41:e84-e85.

9. Treanor JJ, Hayden FG, Vrooman PS, Barbarash R, Bettis R, Riff D, et al. Efficacy and safety of the oral neuraminidase inhibitor oseltamivir in treating acute influenza: a randomized controlled trial. US Oral Neuraminidase Study Group. JAMA. 2000;283:1016-1024.

10. Talavera JO. Investigación clínica I. Diseños de investigación. Rev Med Inst Mex Seguro Soc. 2011;49:53-58.

11. Pérez-Rodríguez M, Palacios-Cruz L, Rivas-Ruiz R, Talavera JO. Investigación clínica XXIV. Del juicio clínico a la ética en investigación en humanos. Rev Med Inst Mex Seguro Soc. 2014;52:666-672.

12. Taveras EM, Marshall R, Sharifi M, Avalon E, Fiechtner L, Horan C, et al. Comparative effectiveness of clinical-community childhood obesity interventions: a randomized clinical trial. JAMA Pediatr. 2017;171:e171325.

13. Rivas-Ruiz R, Talavera JO. Investigación clínica VII. Búsqueda sistemática: cómo localizar artículos. Rev Med Inst Mex Seguro Soc. 2012;50:53-58.

14. Feinstein AR. The architecture of clinical research. Clin Pharmacol Therap. 1970;11.

15. Hulley SB, Cummings SR, Browner WS, Grady DG, Newman TB. Designing clinical research: an epidemiologic approach. EE. UU.: Lippincott Williams \& Wilkins; 2001.

16. Talavera JO, Wacher-Rodarte NH, Rivas-Ruiz R. Investigación clínica III. Estudios de causalidad. Rev Med Inst Mex Seguro Soc. 2011;49:289-294.

17. Portney LG, Watkins MP. Foundations of clinical research. Applications to practice. EE. UU.: F.A. Davis Company; 2015.

18. Bradford-Hill A. The environment and disease: association or causation? Proc R Soc Med. 1965;58:295-300.

19. Zur-Hausen H. Papillomaviruses in the causation of human cancers a brief historical account. Virology. 2009;384:260-265.

20. Narisawa-Saito M, Kiyono T. Basic mechanisms of high-risk human papillomavirus-induced carcinogenesis: roles of E6 and E7 proteins. Cancer Sci. 2007:98:1505-1511.

21. Milner JJ, Rebeles J, Dhungana S, Stewart DA, Summer SC, Meyers MH, et al. Obesity increases mortality and modulates the lung metabolome during pandemic $\mathrm{H} 1 \mathrm{~N} 1$ influenza virus infection in mice. J Immunol. 2015; 194:4846-4859.

22. Kornum JB, Nørgaard M, Dethlefsen C, Due KM, Thomsen RW, Overvad K, et al. Obesity and risk of subsequent hospitalisation with pneumonia. Eur Respir J. 2010;36:1330-1336.

23. Quinn G, Shin CH, Maguire MG, Stone RA. Myopia and ambient lighting at night. Nature. 1999;399:113-114

24. Gwiazda J, Ong E, Held R, Thorn F. Vision: myopia and ambient night-time lighting. Nature. 2000;(404):143-144.

25. Fergusson D, Glass KC, Hutton B, Shapiro S. Randomized controlled trials of aprotinin in cardiac surgery: could clinical equipoise have stopped the bleeding? Clin Trials. 2005;2:218-229.

26. Vanderpool HY, editor. The ethics of research involving human subjects: facing the $21^{\text {st }}$ century. EE. UU.: University Publishing Group; 1996. 
27. Instituto Nacional de Salud Pública. Encuesta Nacional de Salud y Nutrición de Medio Camino 2016. Informe final de resultados. México: Instituto Nacional de Salud Pública/Secretaría de Salud; 2016.

28. Bertado-Cortés B, Villamil-Osorio L, Carrera-Pineda R, Martínez-Cortés C, Guerrero-Cantera J. Características clínicas y sociodemográficas de los pacientes con esclerosis múltiple. Rev Med Inst Mex Seguro Soc. 2016; 54:186-190.

29. Vásquez-Garibay EM, Miranda-Ríos L, Romero-Velarde E, Nuño-Cosío ME, Campos-Barrera L, Nápoles-Rodríguez F, et al. Desmedro, sobrepeso y obesidad durante la transición nutricia en escolares de Arandas, Jalisco, México. Rev Med Inst Mex Seguro Soc. 2018;56:6-11.

30. Keller AC, Knaub LA, Miller MW, Birdsey N, Klemm DJ, Reusch JE. Saxagliptin restores vascular mitochondrial exercise response in the Goto-Kakizaki rat. J Cardiovasc Pharmacol. 2015;65(2):137-147.

31. Del Prato S, Fleck P, Wilson C, Chaudhari P. Comparison of alogliptin and glipizide for composite endpoint of glycated haemoglobin reduction, no hypoglycaemia and no weight gain in type 2 diabetes mellitus. Diabetes Obes Metab. 2016;18:623-627.

32. Kaur K, Likar N, Dang A, Kaur G. Efficacy and safety of canagliflozin among patients with type 2 diabetes mellitus: a systematic review and meta-analysis. Indian J Endocrinol Metab. 2015;19:705-721.

33. Castelán-Martínez OD, Jiménez-Méndez R, Rodríguez-Islas $F$, Fierro-Evans M, Vázquez-Gómez BE, Medina-Sansón A, et al. Hearing loss in Mexican children treated with cisplatin. Int J Pediatr Otorhinolaryngol. 2014;78:1456-1460.

34. Rivas-Ruiz R, Guzmán-Cabañas JM, Párraga-Quiles MJ, Ruiz-González MD, Huertas-Muñoz MD, Alvarez-Marcos R, et al. Utility of the clinical risk index for babies (CRIB) as a predictor of hospital death and intraventricular hemorrhage in very low birth weight and extremely low birth weight neonates. An Pediatr (Barc). 2007;66:140-145.

35. Granados-García V, Sánchez-García S, Ramírez-Aldana R, Zúñiga-Trejo C, Espinel-Bermúdez MC. Costos por hospitalización de adultos mayores en un hospital general regional del IMSS. Rev Med Inst Mex Seguro Soc. 2018;56:S65-S70

36. Barquera S, Campos-Nonato I, Aguilar-Salinas C, López-Ridaura R, Arredondo A, Rivera-Dommarco J. Diabetes in Mexico: cost and management of diabetes and its complications and challenges for health policy. Global Health. 2013;9:3.

37. González-Canudas J, Iglesias-Chiesa JM, Romero-Antonio Y, Chávez-Cortes C, Gay-Molina JG, Rivas-Ruiz R. Cost-effectiveness in the detection of influenza H1N1: clinical data versus rapid tests. Rev Panam Salud Publica. 2011;29:1-8.

38. Castelán-Martínez OD, Hernández-Carbajal E, Contreras-García CE, Ojeda-Luna NG, Rivas-Ruiz R. Eficacia del tratamiento ambulatorio de la neumonía adquirida en la comunidad: revisión sistemática y metaanálisis. Rev Med Inst Mex Seguro Soc. 2016;54:128-136.

39. Miller ME, Williamson JD, Gerstein HC, Byington RP, Cushman WC, Ginsberg $\mathrm{HN}$, et al. Effects of randomization to intensive glucose control on adverse events, cardiovascular disease, and mortality in older versus younger adults in the ACCORD Trial. Diabetes Care. 2014;37:634-643.

40. Beddhu S, Rocco MV, Toto R, Craven TE, Greene T, Bhatt U, et al. Effects of intensive systolic blood pressure control on kidney and cardiovascular outcomes in persons without kidney disease: a secondary analysis of a randomized trial. Ann Intern Med. 2017;167:375-383.

41. Woodward-Lopez G, Gosliner W, Au LE, Kao J, Webb KL, Sagatov RD, et al. Community characteristics modify the relationship between obesity prevention efforts and dietary intake in children: the Healthy Communities Study. Pediatr Obes. 2018;13:46-55.

42. Schauer PR, Bhatt DL, Kirwan JP, Wolski K, Brethauer SA Navaneethan SD, et al. Bariatric surgery versus intensive medical therapy for diabetes: 3-year outcomes. N Engl J Med. 2014;370:2002-2013.

43. Wilson PW, D'Agostino RB, Levy D, Belanger AM, Silbershatz H, Kannel WB, et al. Prediction of coronary heart disease using risk factor categories. Circulation 1998;97:1837-1847.

44. Peacock WF, De-Marco T, Fonarow GC, Diercks D, Wynne J, Apple FS, et al. Cardiac troponin and outcome in acute heart failure. N Engl J Med. 2018;358:2117-2125.

45. Rivas-Ruiz R, Clark P, Talavera JO, Huitrón G, Tamayo JA, Salmerón J. Bone speed of sound throughout lifetime assessed with quantitative ultrasound in a Mexican population. J Clin Densitom. 2015;18:68-75.

46. Herzog JI, Schmahl C. Adverse childhood experiences and the consequences on neurobiological psychosocial, and somatic conditions across the lifespan. Front Psychiatry. 2018;9:420.

47. Riglin L, Collishaw S, Thapar AK, Dalsgaard S, Langley K, Smith GD, et al. Association of genetic risk variants with attention -deficit/hyperactivity disorder trajectories in the general population. JAMA Psychiatry. 2016;73:1285-1292.

48. Gómez-Coronado N, Sethi R, Bortolasci CC, Arancini L, Berk M, Dodd S. A review of the neurobiological underpinning of comorbid substance use and mood disorders. J Affect Disord. 2018;241:388-401. 